\title{
Effect of thermal buoyancy on flow pattern from a pair of side-by-side confined triangular cylinders
}

\author{
H. Laidoudi and M. Bouzit
}

Laboratoire des Sciences et Ingénierie Maritime (LSIM), Faculté de Génie Mécanique, USTO-MB, BP 1505, El-Menaouer, Oran, 31000, Algérie

Received: 12 April 2018

Revised: 13 May 2018

Accepted: 01 July 2019

DOI: https://doi.org/10.3329/bjsir.v55i1.46727

\begin{abstract}
The effects of ax ial and radial thermal buoyancy on fluid flow and mixed convection heat transfer from a pair of identical triangular cylinders in side-by-side arrangement confined within a straight channel. The numerical simulations are carried out by solving continuity, momentum and energy equations using the commercial code ANSYS-CFX. The obtained results are presented and discussed within the range of following conditions: Richardson number $R i=0$ to 2 , Reynolds $R e=20$, and Prandtl number $\operatorname{Pr}=1$ at fixed value of blockage ratio $\beta=0.2$. The main results are depicted in terms of streamline and isotherm contours to analyze the fluidic and energetic behaviors. The total drag coefficient and average Nusselt number are also computed. Moreover, a simple correlation indicating the variations of drag coefficient and average Nusselt number versus Richardson number are also provided. It was found that for axial effect of thermal buoyancy, increase in buoyancy strength enhances the heat transfer rate for both cylinders. In other hand, for radial effect, increase in buoyancy strength increases the heat transfer rate of down cylinder and it is reduced for the upper cylinder.
\end{abstract}

Keywords: Axial buoyancy effect; Radial buoyancy effect; Two triangular cylinders; Heat transfer; Drag coefficient

\section{Introduction}

The analyze of the fluid flow and heat transfer over confined bluff bodies of different cross-sectional forms is one of most classical problem intensively encountered for a long time. The study of this problem is the principal step that provides some important parameters used for the design and development of such mechanical and electrical products such as heat exchangers of high performance, electronic cooling systems, devices of food treatment, etc. It was found that when the flow passes around a confined obstacle a wake and flow separation appear behind the obstacle and the form of the wake depends on several parameters such as velocity of flow, fluid proprieties and the geometrical configuration (the ratio of confinement, cross-sectional form of obstacle and the number of obstacles). Consequently, the flow structure behind a two obstacle cylinders is totally different from that observed behind one cylinder due to reciprocal interactions of wakes behind the cylinders. Besides, the structure of flow even becomes more complicated when the wakes are properly influenced by thermal buoyancy. Based on dimensionless parameters, the thermal buoyancy strength is brought out by increasing gradually the Richardson number $R i=G r / R e^{2}, G r$ being the Grashof number and $R e$ being the Reynolds number. So the Richardson expression provides the strength quantity of natural convection over forced convection.

Recently there are some works studied the fluid flow and heat transfer around a confined cylinder under the axial and radial effects of thermal buoyancy. For axial influence of thermal buoyancy, there are two different effects of thermal buoyancy (aiding and opposing effects). Chatterjee and Mondal (2014) provided through numerical simulations the phenomena of the suppression of flow separation behind a singular cylinder (square and circular form) by aiding thermal buoyancy. Gupta et al. (2017) studied the effect of aiding buoyancy on steady mixed convection heat transfer from one triangular cylinder immersed in power-law fluids in unconfined vertical domain. 
Two different configurations of cylinder are studied; one when the base of the cylinder is facing the flow and the other when the vertex is facing the flow. This study showed that the flow separation behind the cylinder is more pronounced when vertex of triangular facing the flow. Laidoudi and Bouzit (2016) investigated numerically the effects of aiding and opposing thermal buoyancy on downward flow around a circular cylinder confined within vertical channel. The computational results showed that the adding buoyancy enhanced the heat transfer rate; meanwhile the opposing effect reduces the heat transfer rate.

For the radial effect of thermal buoyancy, Sukesan and Dhiman (2014) carried out a numerical investigation of radial buoyancy effect on fluid flow heat transfer from a semi-circular cylinder confined in horizontal channel for steady laminar regime. It was found that flow around the cylinders became asymmetric with gradual increase in the value of radial thermal buoyancy. Laidoudi and Bouzit (2017a) introduced the radial effect of buoyancy on shear-thinning fluids.

Based on the above researches, it is clear that there is no reported work on the effect of thermal buoyancy on fluid flow and heat transfer around a confined two triangular cylinders placed in side-by-side arrangement. Therefore, this paper tries to fill gap in literature. Accordingly, the purpose of present work is to numerically analyze the axial and radial effects of thermal buoyancy on fluid flow and heat transfer around a two triangular cylinders placed in side-by-side arrangement. The obtained results are presented and discussed for the range of these conditions: $R i=0$ to 2 at fixed $\operatorname{Re}=20$ and $\operatorname{Pr}=1$

\section{Problem statement and governing equations}

The problem studied in this paper is schematically illustrated in Figure 1. Two identical heated triangular cylinders with sides $d$ are assumed to place symmetrically in side-by-side arrangement inside of a long two-dimensional channel. The spacing $\mathrm{S}$ showed between the cylinders as well as between cylinders and channel walls are fixed with one width of cylinder $(S=d)$. The upstream and downstream distances of computational domain are gives respectively by the values $L_{u}$ $=10 d$ and $\mathrm{L}_{d}=15 d$, these values are chosen based on our previous work as Laidoudi and Bouzit (2017b). It is also assumed that the flow enters the channel with constant free stream of uniform velocity uin and temperature Tin, and passes the cylinders whose surfaces are fixed temperature $\mathrm{T}_{w}$. the blockage ratio of the computational domain is given as: $\beta$ $=d / H=0.2$. It is also considered that the flow is steady laminar regime with constant thermo-physical proprieties, the thermal buoyancy forces are defined with the aspect of the Boussinesq approximation. For the axial case Figure 1, (a), (a)

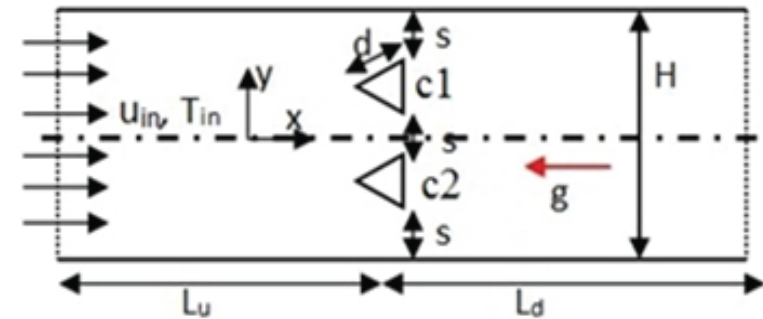

(b)

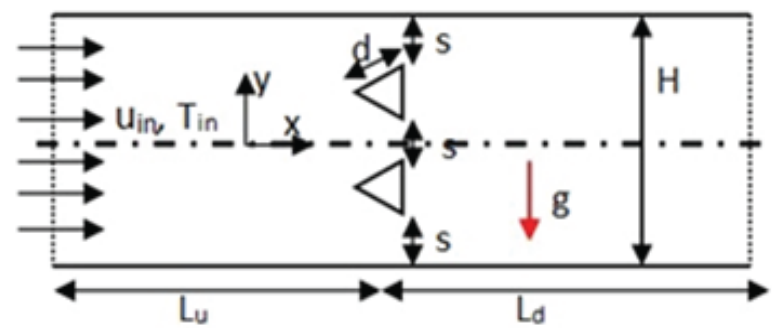

Fig. 1. Schematic diagram of domain (a) Axial buoyancy effect (b) Radial buoyancy effect

the buoyancy force is considered in $\mathrm{x}$-direction aiding the flow direction. For the radial case Figure 1, (b), the buoyancy force is assumed in y-direction crossing the flow direction. The modeling equations of this problem are given by conservation equations of mass, momentum and energy which can be expressed by these dimensionless equations:

$\frac{\partial u}{\partial x}+\frac{\partial v}{\partial y}=0$

- Momentum

(a) For axial buoyancy effect

$$
\begin{gathered}
\frac{\partial u u}{\partial x}+\frac{\partial u v}{\partial y}=-\frac{\partial p}{\partial x}+\frac{1}{R e}\left(\frac{\partial^{2} u}{\partial x^{2}}+\frac{\partial^{2} u}{\partial y^{2}}\right)+R i \theta^{\prime} \\
\frac{\partial u v}{\partial x}+\frac{\partial v v}{\partial y}=-\frac{\partial p}{\partial y}+\frac{1}{R e}\left(\frac{\partial^{2} v}{\partial x^{2}}+\frac{\partial^{2} v}{\partial y^{2}}\right),
\end{gathered}
$$

(b) For radial buoyancy effect

$$
\begin{aligned}
& \frac{\partial u u}{\partial x}+\frac{\partial u v}{\partial y}=-\frac{\partial p}{\partial x}+\frac{1}{\operatorname{Re}}\left(\frac{\partial^{2} u}{\partial x^{2}}+\frac{\partial^{2} u}{\partial y^{2}}\right), \\
& \frac{\partial u v}{\partial x}+\frac{\partial v v}{\partial y}=-\frac{\partial p}{\partial y}+\frac{1}{\operatorname{Re}}\left(\frac{\partial^{2} v}{\partial x^{2}}+\frac{\partial^{2} v}{\partial y^{2}}\right)+\operatorname{Ri\theta }
\end{aligned}
$$


- $\quad$ Energy

$$
\frac{\partial u \theta}{\partial x}+\frac{\partial v \theta}{\partial y}=\frac{1}{\operatorname{PrRe}}\left(\frac{\partial^{2} \theta}{\partial x^{2}}+\frac{\partial^{2} \theta}{\partial y^{2}}\right)
$$

where $\mathrm{u}$, and $\mathrm{v}$ are the dimensionless velocity components along $\mathrm{x}$ - and $\mathrm{y}$-directions of a Cartesian Coordinate system, respectively, $P$ is the dimensionless pressure and $R e=\left(u_{\max }\right.$ $d / \eta)$ is the Reynolds number based on the cylinder dimension. $R i=\left(G r / R e^{2}\right)$ is the Richardson number. $G r=g$ $\left.\beta\left(T_{w}-T_{i n}\right) d^{3} / \eta^{2}\right)$ is the Grash of number with $g$ and $\beta$ being the gravitational acceleration and volumetric expansion coefficient, $\theta$ is the dimensionless temperature and $\operatorname{Pr}=\eta / \alpha$ is the Prandtl number. The fluid properties are described by the density $\rho$, kinematic viscosity $\eta$ and thermal diffusivity $\alpha$. The dimensionless variables are defined as:

$$
\begin{aligned}
& u=\frac{\bar{u}}{u_{i n}}, v=\frac{\bar{v}}{u_{i n}}, x=\frac{\bar{x}}{d}, y=\frac{\bar{y}}{d} . \\
& p=\frac{\bar{p}}{\rho u_{i n}^{2}}, \theta=\frac{\left(T-T_{i n}\right)}{T_{w}-T_{i n}} .
\end{aligned}
$$

The physical boundary conditions of this study can be written as follows:

- At the inlet of channel a uniform velocity profile for laminar flow with a constant temperature, this is can be expressed as:

$u=1, v=0, \theta=0$.

- On the surfaces of the triangular cylinders: The standard no-slip condition is used fluid flow and constant temperature $\mathrm{T}_{w}$.

$u=0, v=0, \theta=1$.

- At the channel walls, the usual no-slip condition for flow and adiabatic condition for energy are used.

$u=0, v=0$, Adiabatic.

- At the outlet Neumann boundary condition for field variables is employed:

$$
\frac{\partial u}{\partial x}=0, \frac{\partial v}{\partial x}=0, \frac{\partial \theta}{\partial x}=0 .
$$

Overall drag coefficient was mathematically defined as:

$$
C_{D}=\frac{2 F_{D}}{\rho u_{i n}^{2} d},
$$

$\mathrm{F}_{D}$ is the total drag force on the surface of the cylinder.

The local Nusselt number on the surface of cylinder was evaluated for constant wall temperature as:

$$
N u_{l}=\frac{h d}{k}=-\frac{\partial \theta}{\partial n_{s}}
$$

where $h$ and $n_{s}$ are: the local surface heat transfer coefficient and the normal direction to the cylinder surface. These local values on entire surface were then averaged to obtain the average Nusselt number of circular cylinder.

$$
N u=\frac{1}{S} \int_{s} N u_{l} d s
$$

\section{Numerical methodology}

The conservation equations shown above subjected to aforementioned boundary conditions are solved numerically using a finite volume method adopted in CFD (Computational Fluid Dynamic) solver called ANSYS-CFX.

The package Gambit has been used to create geometry of computational domain. 204654 elements of triangular cells with non-uniform spacing have been created. In order to get accurate results of the interaction between flow and wall of cylinders, the mesh points have a concentration distribution close to cylinders. The methodology of getting mesh is the same used in our previous works (Laidoudi and Bouzit, 2017c).

\section{Results and discussion}

Figure 2 and Figure 3 show representative streamline contours around the cylinders under the effects of axial and radial buoyancy respectively at fixed values of Reynolds and Prandtl numbers $R e=20$ and $\operatorname{Pr}=1$. From Figure 2, it is observed that the flow structure is perfectly symmetrical, and two recirculation bubbles appeared behind each cylinder, the length of bubbles is seen to be reduced with gradual increase in the Richardson number. Moreover, two identical counter rotating regions are observed in downstream region on the channel walls. From the Figure 3, it is shown that the flow starts to loss its symmetric when the radial effect of buoyancy is added. The degree of asymmetry of flow structure increases with gradual increase in Richardson number. Moreover, the recirculation bubbles behind the cylinders are also influenced by the radial buoyancy effect; they interact in upward direction with gradual increase in buoyancy strength. Furthermore, the radial effect of thermal buoyancy creates two different kinds of recirculation zones, the first one is situated in upstream region above the cylinders and the second is in downstream region under the cylinders.

Figures 4 and 5 depict some representative contours of the isotherms for different values of Richardson number at fixed value of Reynolds number $R e=20$ and Prandtl number of $\mathrm{Pr}$ $=1$. Figure 4 is for axial buoyancy effect meanwhile the 
(a) $\mathrm{Ri}=0$

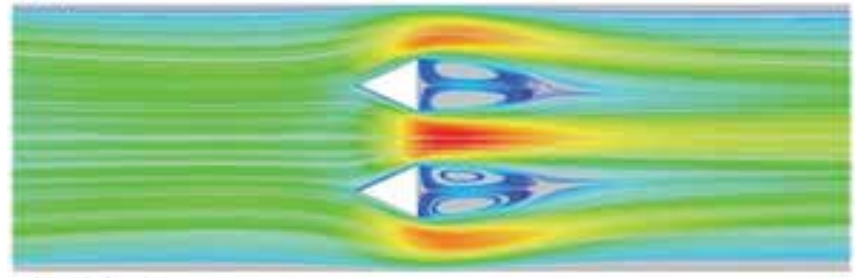

(b) $\mathrm{Ri}=1$

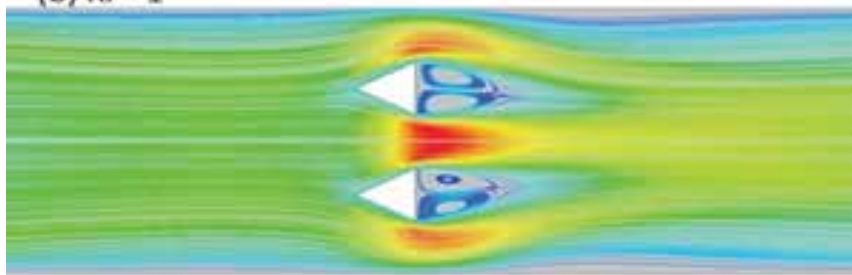

\section{(c) $\mathbf{R} \mathbf{j}=2$}

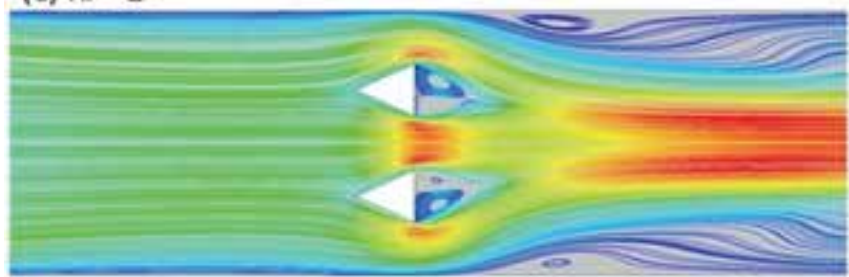

Fig. 2. Streamlines around cylinders for $\operatorname{Re}=20$ and $P r=$ 1 under axial buoyancy effect

(a) $\mathrm{Ri}=0.5$

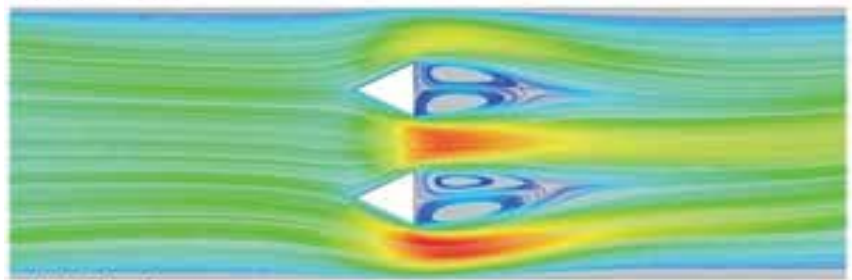

(b) $\mathrm{Ri}=1$

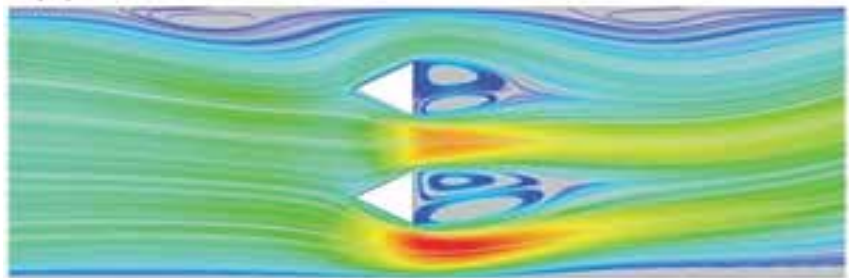

(c) $\mathrm{Ri}=2$

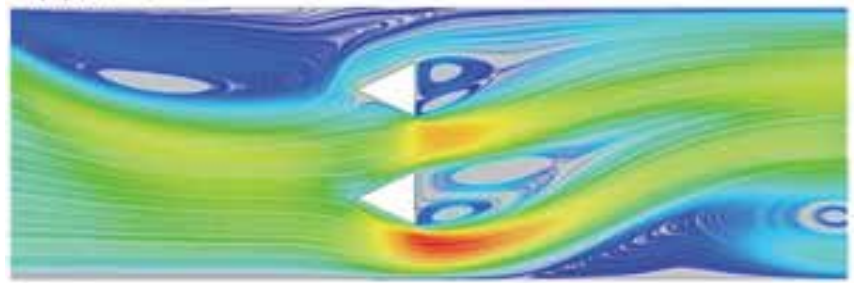

Fig. 3. Streamlines around cylinders for $\operatorname{Re}=\mathbf{2 0}$ and $\mathrm{Pr}=$ 1 under radial buoyancy effect (a) $\mathrm{Ri}=0$
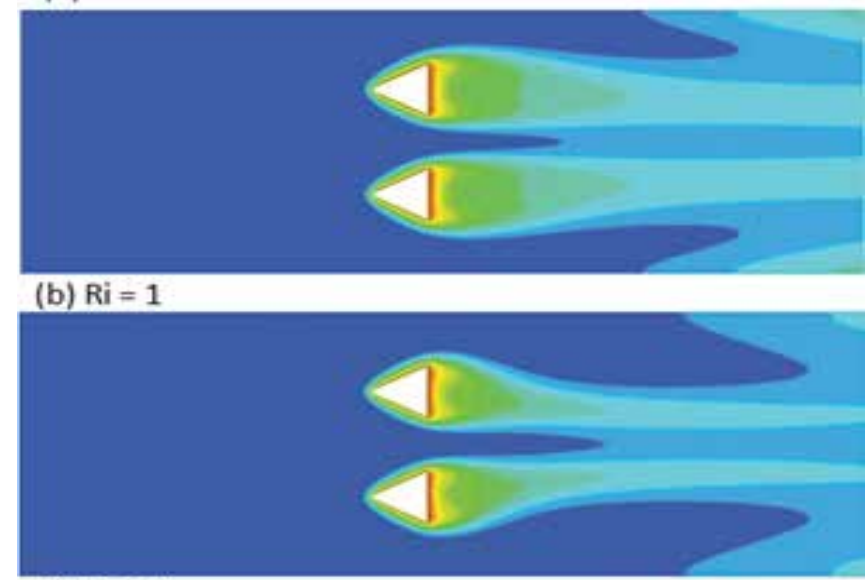

(c) $\mathrm{Ri}=2$

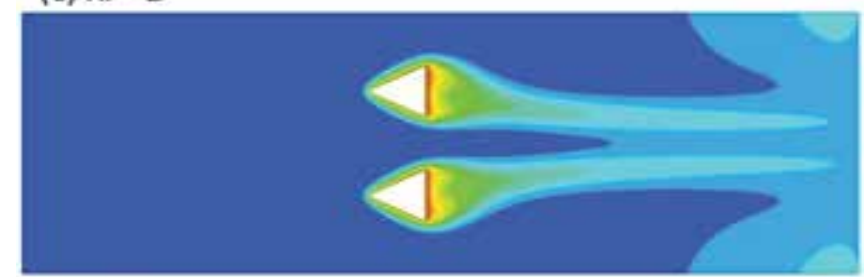

Fig. 4. Isotherms around cylinders for $\operatorname{Re}=20$ and $P r=1$ under axial buoyancy effect

(a) $\mathrm{Ri}=0.5$
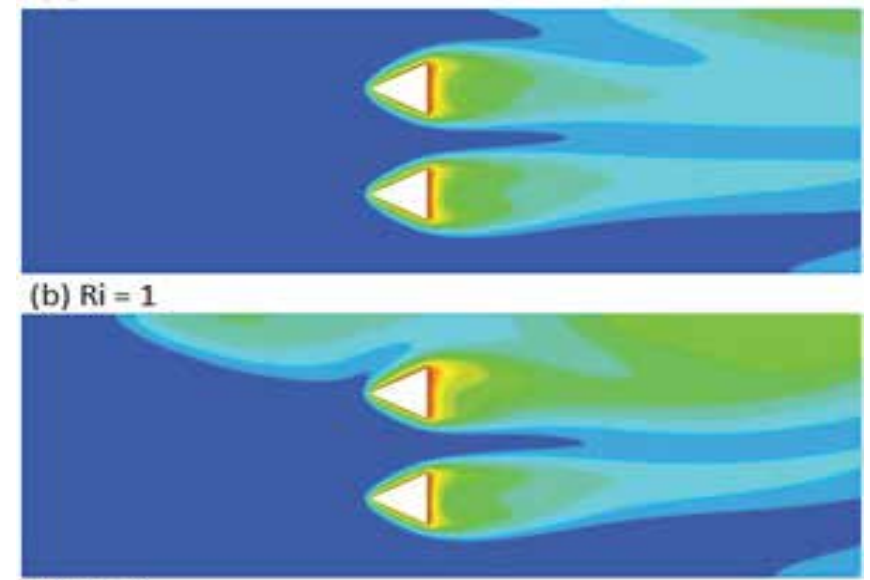

(c) $\mathrm{Ri}=\mathbf{2}$

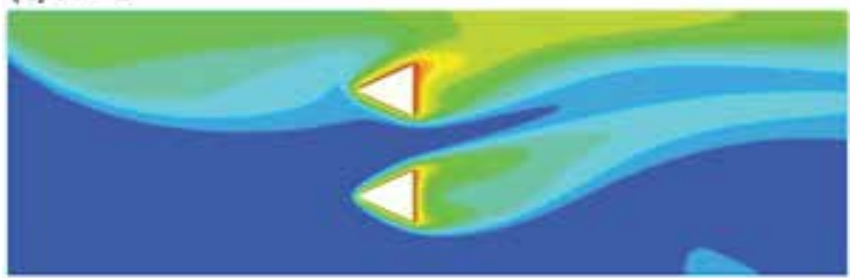

Fig. 5. Isotherms around cylinders for $\mathrm{Re}=\mathbf{2 0}$ and $\mathrm{Pr}=\mathbf{1}$ under radial buoyancy effect 
Figure 5 is for radial effect. As streamlines, the isotherms are symmetrical under the axial buoyancy effect but they are asymmetrical for radial effect. It is known that crowding isotherms indicate high temperature gradient and consequently higher heat transfer rate. Based on this information, it is observed that crowding of the isotherms increases systematically with gradual increase in Richardson number for axial effect of the buoyancy but this behavior is seen to be changed for upper cylinder under the radial effect of thermal buoyancy.

Figures 6 and 7 present the variations of drag coefficient of both cylinders versus Richardson number at fixed $R e=20$ and

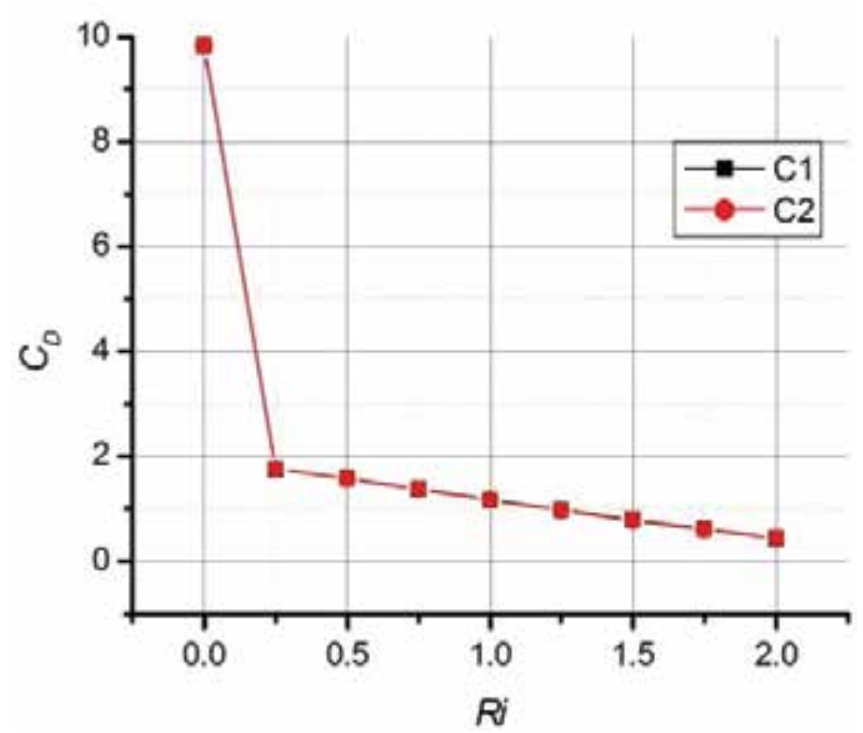

Fig. 6. Variation of drag coefficient under the axial buoyancy effect at $\operatorname{Re}=20$ and $\operatorname{Pr}=1$

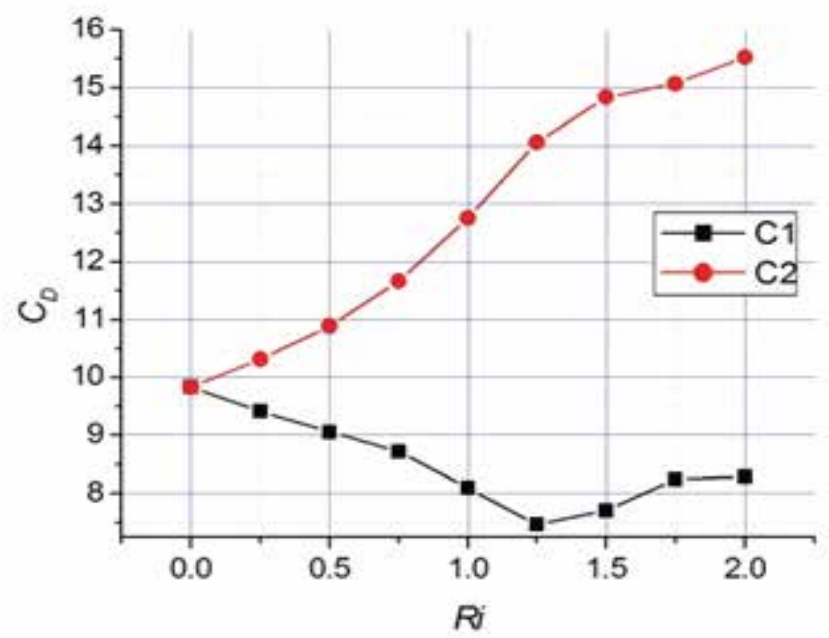

Fig. 7. Variation of drag coefficient under the radial buoyancy effect at $\operatorname{Re}=20$ and $\operatorname{Pr}=1$
$\operatorname{Pr}=1$ under the effect of axial and radial thermal buoyancy. The potted graphs of Figure 6 show that the total drag coefficient of both cylinders decreases with increase Reynolds number, this is due to velocity of fluid particles which is increased behind the cylinders in the flow direction and consequently the recirculation zones behind the cylinders decreased which indicate that the difference of pressure exerted on cylinder surfaces is decreased. The plotted graphs of the Figure 7 indicate that the total drag coefficient of upper (C1) cylinder increases gradually with increase in Richardson number meanwhile a different dependence on Richardson number is observed for the down (C2) cylinder, this is also due to radial effect of buoyancy which created a radial velocity of fluid particles behind cylinders.

The following simple correlation was obtained for total drag coefficient for the range of Richardson number limited between 0.5 and 2 at $R e=20$ and $P r=1$ under the axial effect of thermal buoyancy.

$\mathrm{C}_{\mathrm{D}}=1.9374-0.76393 \mathrm{Ri}$

Both Figure 8 and 9 show the variation of average Nusselt number of both cylinders with Richardson number at $R e=20$ and $\operatorname{Pr}=1$ under the axial and radial buoyancy effect respectively. Figure 8 depicted that the $\mathrm{Nu}$ increases with increase in Richardson number for both cylinders but Figure 9 shows that the average Nusselt number of upper cylinder increase gradually with Richardson number meanwhile the down cylinder shows an inverse evolution with Richardson number. Also a simple correlation of average Nusselt number versus Richardson at $\operatorname{Re}=20$ and $P r=1$ is added only for the axial effect of thermal buoyancy.

$\mathrm{Nu}=2.30675+0.13233 \mathrm{Ri}$

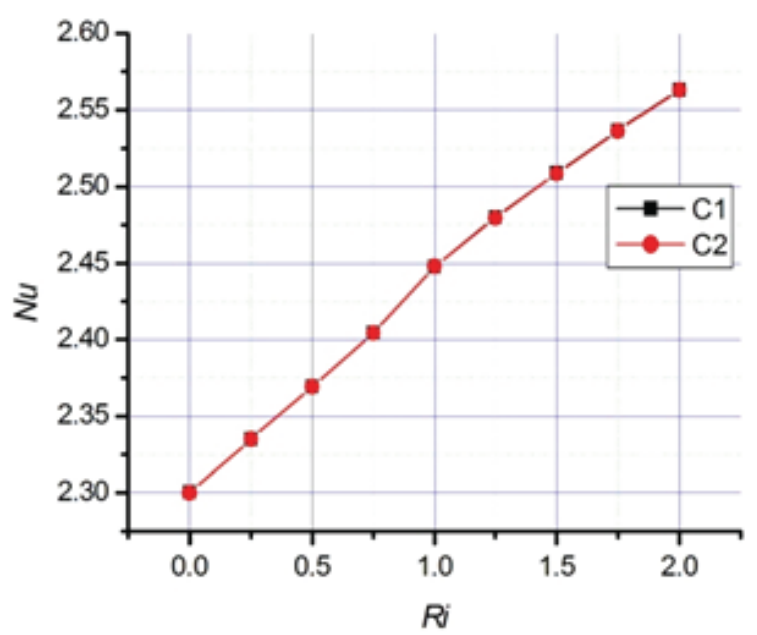

Fig. 8. Variation of Nusselt number under the axial buoyancy effect at $\operatorname{Re}=20$ and $\operatorname{Pr}=1$ 


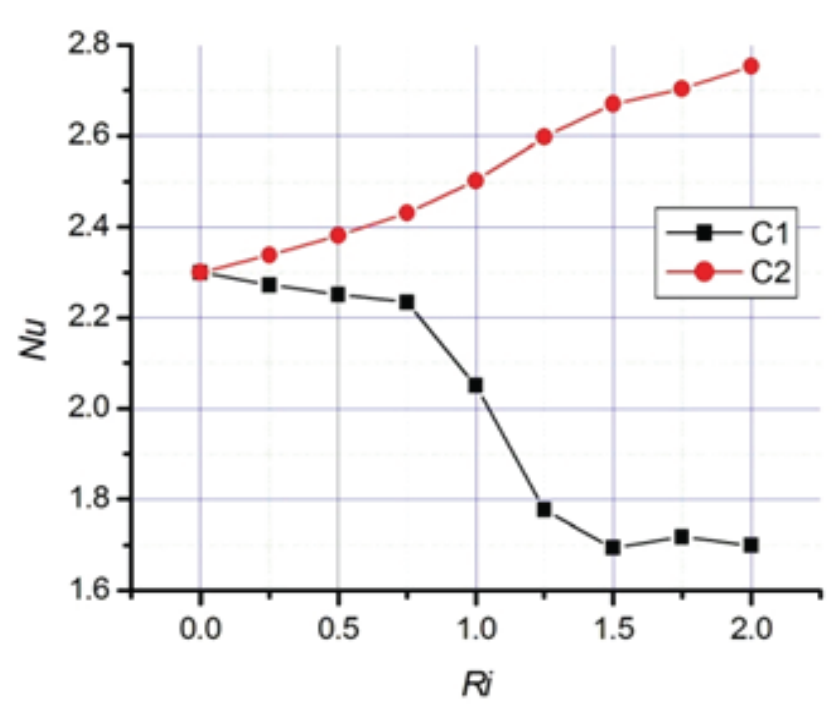

Fig. 9. Variation of Nusselt number under the radial buoyancy effect at $\operatorname{Re}=20$ and $\operatorname{Pr}=1$

\section{Conclusion}

Two-numerical simulations are carried out to analyze the effect of axial and radial thermal buoyancy on fluid flow and heat transfer over a pair of triangular cylinders placed in side-by-side arrangement. The investigation is done for the range of following conditions: Richardson number, $R i=0$ to 2 at fixed value of Reynolds number of $R e=20$, Prandtl number of $\operatorname{Pr}=1$ and blockage ratio of $\beta=0.2$.

The main finding of this research can be summarized as follow:

1. For axial effect of thermal buoyancy: the gradual increase in Richardson number reduces the size of recirculation zones behind the cylinders and decreases the stability of the flow in downstream zone. Also, it is found that increase in the value of Richardson number gradually decreases the total drag coefficient of cylinders and improves the average Nusselt number of cylinders.

2. For radial effect of thermal buoyancy: increase in Richardson number creates an asymmetrical flow around the cylinder and two new sort of counter-rotating zones appear in upper part of upstream zone and down part of downstream zone respectively. Moreover, increase in the radial effect buoyancy increases the total drag coefficient and average Nusselt number of down cylinder. On other hand, it decreases the total drag coefficient and average Nusselt number of upper cylinder.

\section{References}

Chatterjee D and Mondal B (2014), Control of flow separation around bluff obstacles by superimposed thermal buoyancy, J. Heat and mass transfer 72: 127-138. DOI: $10.1016 /$ j.ijheatmasstransfer. 2014.01 .013

Gupta SK, Ray S and Chatterjee D (2017), Steady mixed convection in power-law fluids from a heated triangular cylinder, J. Heat Transfer Engineering 20: 1-20. DOI: 10.1080/01457632.2017.1357773

Laidoudi H and Bouzit M (2016), Mixed convection in Poiseuille fluid from an asymmetrically confined heated circular cylinder, J. Thermal Science 00: 172-172. DOI: 10.2298/TSCI160424172L

Laidoudi H and Bouzit M (2017a), Mixed convection heat transfer from confined tandem circular cylinders in cross-flow at low Reynolds number, Mechanics 23: 522-527. DOI: 10.5755/j01.mech.23.4.15258

Laidoudi H and Bouzit M (2017b), Suppression of flow separation of power-law fluids flow around a confined circular cylinder by superimposed thermal buoyancy, Mechanika 23: 220-227. DOI: 10.5755/j01.mech.23.2.14342

Laidoudi H and Bouzit M (2017c), The effects of aiding and opposing thermal buoyancy on downward flow around a confined circular cylinder, J. Periodica Polytechnica Mechanical Engineering 62(1): 42-50. DOI: 10.3311/PPme.11382

Sukesan K and Dhiman A K( 2014), Laminar mixed convection in a channel with a built-in semi-circular cylinder under the effect of cross-buoyancy, International Communications in Heat and Mass Transfer 58: 25-32. DOI: 10.1016/j. icheatmasstransfer.2014.08.025 